\title{
Impact Des Réformes Sur La Compétitivité De La Production De Riz En Côte d'Ivoire Et Analyse De Sensibilité
}

\author{
Achy Landry \\ Université Félix Houphouët Boigny d’Abidjan
}

doi: 10.19044/esj.2016.v12n33p149 URL:http://dx.doi.org/10.19044/esj.2016.v12n33p149

\begin{abstract}
The rice landscape since independence from the Ivory Coast to the present, is characterized by a series of government policies for the sector. The latest is the Revised National Strategy for Development of the rice sector (NRDS) 2012 - 2020.

The central objective of this study is to analyze the impact on the competitiveness of producers, strategic guidelines defined by the NRDS.

Our results indicate that it will generally have a positive effect on the competitiveness of production systems. But we find her, the existence of unfavorable conditions for the cultivation of upland rice system.

Furthermore, a sensitivity analysis completing the foregoing analysis is performed. The results of this simulation show that: i) the increase in yield was identified as the only factor able to improve both financial profitability, external competitiveness (comparative advantage), reducing at the same time support producers; ii) the competitiveness of local rice increased with the overvaluation of the CFA.

All these previous analyzes using the Policy Analysis Matrix (MAP) as an analytical model.
\end{abstract}

Keywords: Revised National Rice Development Strategy ; competitiveness ; production system ; sensibility analysis ; Policy Analysis Matrix

\section{Résumé}

Le paysage rizicole depuis l'indépendance de la Côte d'Ivoire à nos jours, se caractérise par une succession de politiques gouvernementales en faveur de cette filière. La plus récente est la Stratégie Nationale Révisée de Développement de la filière Riz (SNDR) 2012 - 2020. L’objectif central de cette étude est d'analyser l'impact sur la compétitivité des producteurs, des orientations stratégiques définies par cette SNDR. 
Nos résultats indiquent que cette dernière aura en général, un effet positif sur la compétitivité des systèmes de production. Mais, nous trouvons avec elle, l'existence de conditions défavorables pour la culture du système de riz pluvial.

Par ailleurs, une analyse de sensibilité complétant l'analyse précédente est effectuée. Les résultats obtenus de cette simulation montrent que: i) l'accroissement du rendement a été identifié comme le seul facteur capable d'améliorer à la fois la rentabilité financière, la compétitivité extérieure (avantage comparatif), en réduisant par la même occasion le soutien aux producteurs ; ii) la compétitivité du riz local augmente avec la surévaluation du FCFA.

Toutes ces précédentes analyses utilisent la Matrice d’Analyse Politique (MAP) comme modèle d'analyse.

Mots clés: Stratégie Nationale Révisée de Développement de la filière Riz ; compétitivité ; système de production; analyse de sensibilité ; Matrice d’Analyse Politique

\section{Introduction}

La politique menée par les Gouvernants en matière de riz peut s’analyser sur trois périodes :

- $\quad$ De 1960 à 1977, caractérisée par une politique volontariste de l'Etat sur l'ensemble de la filière et son aboutissant, c'est la création de la SODERIZ.

- $\quad$ De 1978 à 1995, la filière riz est confiée successivement à plusieurs structures. A cette période, nous avons toujours l'intervention de l'Etat qui a diminué au fil des années vu qu'en 1982, il y a eu une chute drastique du riz importé au niveau international. En effet, le riz local devenant moins compétitif par rapport au riz importé, le gouvernement abandonne peu à peu sa politique d'autosuffisance en riz. Par la suite, Il abandonne aussi sa politique de péréquation des prix qui débouche sur la dissolution de la CGPP en mars 1995. Ce qui consacre la libéralisation totale de la filière riz.

- De 1996 à nos jours, nous avons une politique de recherche d’autosuffisance alimentaire générale qui n’est pas spécifiquement liée au riz parce qu'il n'existe pas de structure étatique qui a à charge l'ensemble de la filière riz comme à l'époque de la SODERIZ. Mais, il faut remarquer que la mauvaise conjoncture due à la flambée des prix du riz pendant l'année 20072008 et qui a court encore aujourd'hui, a convaincu nos dirigeants de la nécessité de rechercher l'autosuffisance en riz au travers de la Stratégie Nationale Révisée de Développement de la filière Riz (SNDR) 2012 - 2020.

Cette présente Stratégie Nationale Révisée de Développement du Riz (SNDR) est une révision de celle de 2008 pour en corriger les insuffisances 
et a pour ambition d'augmenter la production nationale de riz, soutenable économiquement, socialement et ce, en vue d'une réduction notable des importations dans la consommation nationale de riz et d'augmenter corrélativement les revenus des producteurs nationaux. A cette fin, plusieurs mesures de reformes sont définies par le gouvernement afin d'améliorer la compétitivité de la production de riz local.

Mais, cette stratégie est-elle suffisamment ambitieuse pour modifier la rentabilité actuelle et la structure des incitations agricoles actuelles des systèmes de production du riz local ?

Pour y répondre, cette étude vise à analyser l'impact sur la compétitivité des producteurs des orientations stratégiques définies par la SNDR. Ensuite, une analyse de sensibilité complétant l'analyse précédente sera effectuée pour apprécier les changements de la compétitivité des producteurs par rapport à certaines variables clefs. Pour se faire, plusieurs modèles peuvent servir à faire des simulations.

Avec la Matrice d’Analyse de Politiques (MAP), Houndekon (1996) réalise un ensemble de simulations. Ses résultats montrent une amélioration de la compétitivité, suite à la dévaluation, à la hausse des prix internationaux. Cependant, il n’a pas comparé l'effet de ces différentes variables sur le coût en ressource intérieure afin de déterminer celle qui a le plus grand effet. Sylla (1997) mettra fin à cet handicap, en comparant l'effet sur la compétitivité d'une dévaluation et celui d'une hausse des cours mondiaux. Il utilise à cet effet, en plus de la MAP, une démarche statistique. Il s’agit de la statistique comparative qui consiste à faire varier un seul facteur tandis que les autres sont maintenus constants. Koné (1998) utilise cette dernière pour faire son analyse de sensibilité. Son analyse prouve que seul un accroissement du rendement est de nature à améliorer la rentabilité financière et l'avantage comparatif des systèmes de production du coton et du riz. Nous envisageons construire nos simulations à partir des travaux de ces deux précédents auteurs.

\section{Brève présentation de la Stratégie Nationale Révisée de Développement de la filière Riz (SNDR) 2012 - 2020} Vision

La vision de cette Stratégie révisée est de produire de manière compétitive, rentable et durable, du riz de bonne qualité et concurrentiel par rapport au riz importé, pour satisfaire nos besoins nationaux et de constituer un stock de sécurité avec l'opportunité d'exporter le surplus de production en impliquant tous les systèmes de producteurs de riz à savoir le riz pluvial, le riz de bas-fond, le riz irrigué et le riz inondé.

Le corollaire à cette vision est de faire de la production du riz, une activité hautement rémunératrice pour les riziculteurs, capable de contribuer 
efficacement à assurer la sécurité alimentaire et à lutter contre la pauvreté en Côte d'ivoire et dans la sous région.

La Stratégie prévoit de couvrir les besoins nationaux en riz à partir de 2016 avec un niveau de production de 1.900.000 tonnes de riz blanchi pour une consommation estimée pour la même période à 1.800 .000 tonnes de riz blanchi. A l'horizon 2018, il s'agira d'accroître la production locale pour atteindre 2.100.000 tonnes de riz blanchi pour une consommation estimée à environ 1.915.000 tonnes et dégager environ 200.000 tonnes afin de constituer un stock de sécurité.

Tableau 1 : Projections estimées de la Production et de la Consommation en Côte d’Ivoire avec les actions de la stratégie révisée

\begin{tabular}{|c|c|c|c|c|}
\hline Année & Population (Hbts) & $\begin{array}{c}\text { Consommation } \\
\text { (Tonnes) }\end{array}$ & $\begin{array}{c}\text { Production Nationale } \\
\text { (Tonnes) }\end{array}$ & $\begin{array}{c}\text { Déficit /Surplus } \\
\text { (Tonnes) }\end{array}$ \\
\hline 2008 & 20600000 & 1360704 & 604024 & -756680 \\
\hline 2009 & 21,900000 & 1547265 & 628184 & -919081 \\
\hline 2013 & 25800000 & 1628000 & 1432000 & -196000 \\
\hline 2015 & 27500000 & 1737000 & 1891340 & 154340 \\
\hline 2018 & 30300000 & 1915000 & 2112500 & 197500 \\
\hline
\end{tabular}

Source : ONDR, 2012

\section{Impact}

L'impact attendu de la mise en œuvre de la stratégie de développement durable de la riziculture est de permettre à la Côte d'ivoire de couvrir ses besoins de consommation par sa production nationale et de contribuer ainsi au développement de l'économie, au maintien de la paix sociale et à la création de plusieurs nouveaux emplois. Ce qui permettra de contribuer efficacement à la lutte contre la pauvreté dans le pays et dans la sous région ouest africaine.

En situation de croisière, des flux financiers importants seront générés tant en amont auprès des fournisseurs de services (intrants et équipements) qu'en aval avec les stocks de produits finis (riz blanchi) et sous produits (farine basse et briquettes) échangés. Le chiffre d'affaires de la commercialisation du riz blanchi est estimé à 630 milliards de francs CFA en 2016 sur la base d'un prix indicatif de 350 F CFA le kilogramme de riz blanchi.

Les sous produits qui représentent environ 20\% sont valorisés à 126 milliards de francs CFA.

La contribution de la filière riz en termes de taxes et impôts pourrait avoisiner les 56 milliards de francs CFA par an (7,5\% du chiffre d'affaires) nonobstant les flux engrangés en amont au niveau des intrants et équipements.

Les créations d'emplois concerneront tous les segments de la chaîne de valeur du riz notamment au niveau de la production, des services (CGEA, 
transformation, diverses maintenances etc.) et de la distribution. Ainsi, plus de 500.000 emplois directs et 2.000.000 d'emplois indirects seront créés en amont et plus de 100.000 emplois directs et 400.000 d'emplois indirects en aval notamment dans les domaines de la transformation et des services.

Au plan de l'aspect structurant du milieu rural, la stratégie favorisera d'une part la création de richesses relativement bien distribuées, secondairement une nette amélioration des niveaux de vie perceptible par l'accessibilité aux commodités modernes, freinant ainsi sensiblement l'exode rural et d'autre part l'émergence de PMEA dirigés par des cadres ruraux véritables animateurs du développement communautaire et régional.

\section{Impact des réformes gouvernementales sur la compétitivité de la production de riz local \\ Revue de la littérature}

Le modèle de la matrice d'analyse des politiques (MAP) permet de mesurer l'impact des politiques de prix sur la compétitivité et la profitabilité d'une filière et de ses différentes activités (Monke and Pearson, 1989). Mais, ce cadre de la MAP développé par Monke et Pearson (op cit) est complété par un développement de l'analyse de la distorsion des prix apportée par Masters et WinterNelson (1995), qui tient compte de l'évaluation des intrants non échangeables.

Fang et al. (2000), évaluent l'avantage comparatif et la protection des principales cultures agricoles de la Chine à l'aide d'une matrice d'analyse des politiques modifiée (MAP) (conformément à ce qui précède) et des données de 1996 à 1998. Leurs résultats suggèrent que la politique chinoise d'autosuffisance en céréales entraînait des pertes d'efficacité. Par ailleurs, leurs résultats éclairent les changements probables des modèles de commerce agricole en Chine, si l'adhésion à l'Organisation mondiale du commerce (OMC) a lieu. Ils soulignent également la nécessité d'une plus grande productivité des intrants dans la production céréalière pour améliorer sa compétitivité si la Chine maintient sa politique de sécurité alimentaire.

Mohanty et al. (2002), utilisent aussi une approche modifiée de matrice d'analyse des politiques (MAP) pour évaluer l'efficacité de la production de coton dans cinq grands États producteurs de l'Inde. Leurs résultats indiquent que le coton n'est pas produit efficacement dans la deuxième province cotonnière du pays. En outre, ils trouvent que le coton n'est pas la culture la plus efficacement produite dans les quatre autres États; cependant, il y a au moins une culture dans chaque État qui est produite moins efficacement que le coton.

L’année 2007-2008 est marquée par le début de la crise alimentaire mondiale caractérisée par la flambée des prix des denrées alimentaires. Dans le courant de cette crise, des travaux de recherches dans le cadre du projet 
SRAI (Strengthening Regional Agricultural Integration in West Africa) sont conduits dans 7 pays de la sous-région (Bénin, Burkina-Faso, Côte d’Ivoire, Guinée, Mali, Niger et Sénégal) en 2011. Ils ont consisté à la construction de plusieurs MAP afin d’apprécier la rentabilité et la compétitivité de la riziculture ouest africaine depuis la flambée des prix. Les résultats de cette étude montrent qu'avant et après la flambée des prix, la riziculture irriguée, de bas fonds ou pluviale dans les différents pays est demeurée financièrement et économiquement rentable. Dans la majorité des cas (spécifiquement en Côte d'Ivoire), les profits financiers sont supérieurs aux profits économiques indiquant que les producteurs ont été soutenus à travers les programmes de subvention aux intrants mises en œuvre dans la plupart des pays.

En Côte d'Ivoire, Sylla (1997), montre qu’avant la dévaluation, les opérateurs économiques des filières café, cacao et riz étaient plus protégés, financièrement et économiquement moins rentables (sauf la filière cacao) dans la région du centre ouest. Avec la dévaluation, on assiste à une situation contraire où ces opérateurs sont moins protégés, financièrement et économiquement plus rentables.

Par ailleurs, Koné (1998), montre que globalement la sous-filière cotonnière est rentable financièrement et économiquement dans les zones de Boundiali et d'Odienné. Les ratios coût bénéfice financier (RCBF) et de coût en ressources intérieures (CRI) sont respectivement de 0,78 et de 0,89. Cependant, il trouve un résultat contraire pour la sous-filière riz.

En outre, Ouattara (2011), avec aussi la construction de plusieurs MAP, montre que l'activité rizicole est financièrement rentable en Côte d'Ivoire. Les profits financiers par tonne de riz blanc produit en zones forestières et des savanes sont respectivement de 140909 F et 129349 F. Par ailleurs, au vue de la rentabilité économique (97 $005 \mathrm{~F}$ et $115445 \mathrm{~F} /$ tonne de riz blanchi), l'on peut dire que la filière contribue à la croissance économique du pays.

\section{Source des données et modèle d'analyse}

\section{* Source des données et méthode d'échantillonnage}

Dans le cadre du Programme d'Appui Stratégique à la Recherche Scientifique (PASRES), nous avons bénéficié d'un financement en 2012 pour collecter des données primaires auprès de la population cible : les riziculteurs de Côte d'Ivoire. Le choix des zones d'étude aussi bien que la sélection de l'échantillon ont visé prioritairement une bonne représentation de toutes les écologies rizicoles de la Côte d'Ivoire. Un questionnaire à passage unique a permis de recueillir les données socio-économiques des producteurs, celles relatives aux prix et à la gestion des exploitations. 


\section{* Matrice des politiques rizicoles futures et actuelles (2012)}

Pour analyser la politique future rizicole, deux options ont été choisies. Le tableau suivant donne quelques indications sur ces options.

Tableau 2 : Matrice des politiques rizicoles futures et actuelles (2012)

Options

Description des

Incidences institutionnelles

politiques

Option

No 1

Option

No 2

SNDR 20122020 néant

rendre les semences sélectionnées disponibles sur l'ensemble des zones de production;

réhabiliter les 35.000 ha actuellement aménagés et réaliser de nouveaux aménagements afin de passer de 30.000 ha à 45.000 ha d'aménagement pour la pratique du riz irrigué en deux (2) cycles de production annuelle ; améliorer les rendements du riz pluvial d'au moins 30\% ; avoir pour le riz irrigué, un rendement d'au moins cinq (5) tonnes par cycle soit dix (10) tonnes au moins par an de paddy

Source : scénario à partir d’ONDR, 2012

La comparaison de l'option 2 à l'option 1 nous permettra de tirer les conclusions sur l'efficacité des politiques futures relativement aux politiques du gouvernement en 2012, année de déroulement de notre enquête de terrain. Mais, cette comparaison s'appuie sur la matrice d'analyse de politiques comme modèle d'analyse à l'instar des récentes études d'impact de chocs exogène et interne sur la compétitivité des filières agricoles en Afrique de l'Ouest et dans d'autres zones du monde.

\section{* Présentation de la matrice d'analyses de politiques (MAP)}

La MAP se présente comme dans le tableau suivant.

Tableau 3: Présentation de la Matrice d'analyse des politiques

Revenus Coûts des facteurs

Biens échangeables

Prix de marché

Prix de éférence

Ecarts

$\begin{array}{ll}\text { A } & \text { B } \\ \text { E } & \text { F } \\ \text { I } & \text { J }\end{array}$

B

$\mathrm{F}$

Source : Monke, E. A. et Pearson, S.R (1989)
Profits échangeables

C

G

$\mathrm{K}$

$\mathrm{H}$

$\mathrm{L}$

La 1ère ligne de cette matrice permet de mesurer la rentabilité de la filière c'est à dire qu'elle se rapporte aux recettes et aux cours observés sur le marché. Il s'agit des prix de marché réellement perçus par les paysans, les collecteurs, les transformateurs et les commerçants.

La 2ème ligne témoigne de l'efficacité économique des opérateurs de la filière. Pour établir cette ligne, il faut estimer les prix de référence. Pour les biens échangeables, les prix de référence sont les prix de parité 
internationale (prix de parité à l'importation pour les biens importables et prix de parité à l'exportation pour les biens exportables).

En définitive, la 3ème ligne montre les différences entre la rentabilité privée et la rentabilité sociale. Elle correspond aux effets négatifs de la politique et montre les imperfections du marché.

Mais, nous voulons indiquer que c'est une MAP au niveau des producteurs qui est construite pour cette étude.

Connaissant les variables fondamentales (A, B, C, D, E, F, G), les différents indicateurs peuvent être calculés. Le tableau 4 suivant indique les indicateurs utilisés.

1. Rentabilité Financière

2. Ratio Coût-Bénéfice Financier

3. Rentabilité Economique

$[\mathrm{D}=\mathrm{A}-\mathrm{B}-\mathrm{C}]$

4. Coût En Ressources Intérieures

$[\mathrm{RCBF}=\mathrm{C} /(\mathrm{A}-\mathrm{B})]$

5. Ratio Coût-Bénéfice Economique

$[\mathrm{H}=\mathrm{E}-\mathrm{F}-\mathrm{G}]$

6. Transferts

$[\mathrm{CRI}=\mathrm{G} /(\mathrm{E}-\mathrm{F})]$

7. Coefficient Protection Nominal

$[\mathrm{RCBE}=(\mathrm{F}+\mathrm{G}) / \mathrm{E}]$

8. Coefficient Protection Effective

$[\mathrm{L}=\mathrm{I}-\mathrm{J}-\mathrm{K}]$

9. Coefficient De Rentabilité

$[\mathrm{CPN}=\mathrm{A} / \mathrm{E}]$

$[\mathrm{CPE}=(\mathrm{A}-\mathrm{B}) /(\mathrm{E}-\mathrm{F})]$

10. Taux Subvention Producteur

$[\mathrm{TPG}=\mathrm{D} / \mathrm{H}]$

11. Equivalent Subvention Producteur

$[\mathrm{TSP}=\mathrm{L} / \mathrm{E}]$

$[\mathrm{ESP}=\mathrm{L} / \mathrm{A}]$

\section{Résultats des analyses \\ * $\quad$ Riz pluvial (RP)}

Tableau 5 : MAP avec la SNDR pour le système de riz pluvial

Recettes (en Intrants échangeables Domestiques (en

$\begin{array}{llll}\text { FCFA) (en FCFA) } & \text { FCFA) }\end{array}$

Prix du

marché

Prix de

référence

583875

57991

120308

405576

Divergences

984982

55213

117525

812244

$-401107$

2778

2783

$-406668$

$\mathrm{RCBF}=0,23$

$\mathrm{CRI}=0,13$

$\mathrm{CPN}=0,59 \mathrm{NPI}^{1}=$

$\mathrm{RCBE}=0,18 \quad \mathrm{CR}=0,50$

1,05

$\mathrm{CPE}=0,57$

$\mathrm{TSP}=-0,41$

$\mathrm{ESP}=-0,7$

Indicateurs MAP statu quo ou MAP après la crise post- électorale

$\mathrm{RCBF}=0,26 \quad \mathrm{RCBE}=0,2 \quad \mathrm{CRI}=0,14 \quad \mathrm{CR}=0,48 \quad \mathrm{CPN}=0,59 \quad \mathrm{CPE}=0,56 \quad \mathrm{TSP}=$ $-0,41$ ESP $=-0,7$ NPI $=1,05$

Source : calcul de l'auteur

L'analyse de la matrice d'analyse de la politique à travers la mesure de l'efficacité économique du système riz pluvial par l'indicateur CRI, révèle

${ }^{1}$ NPI : Coefficient de protection nominale des intrants échangeables 
qu'en 2012, la production du riz local est compétitive, Il utilisait les ressources domestiques, avec une rationalité économique de $86 \%$, et cet avantage comparatif satisfaisant s'améliore légèrement avec la mise en œuvre de la mesure de politique relative à l'amélioration des rendements. Elle exploite désormais ces ressources domestiques avec une efficacité économique de $87 \%$. Ainsi, ce résultat montre que cette nouvelle stratégie de développement du riz n'influence que peu la compétitivité du système de RP dans la mesure où celui-ci n’engendre pas de dépenses d'aménagement comme avec le système de bas fond. Néanmoins, il a besoin d'être soutenu par un appui technique et une disponibilité en intrants.

Concernant le RCBF qui traduit la capacité d'autofinancement des producteurs de ce système, nous obtenons une nette amélioration de cet indicateur. En effet, avec la mise en œuvre de la SNDR, on s'attend à un $\mathrm{RCBF}=0,23$; alors qu'il était de 0,26 en 2012. Ainsi, avec cette stratégie, nos paysans qui emploient ce système supportent davantage les coûts domestiques tout en restant rentables.

Le CPN (coefficient de protection nominal) permet d'établir le rapport entre le prix de marché et le prix économique du paddy local. Pour ce système, ce coefficient est inférieur à $1(\mathrm{CPN}=0.59)$. Cela traduit le fait que le prix du riz local observé sur le marché domestique est très inférieur à celui du marché international. Ainsi, les producteurs perçoivent des revenus inférieurs à ceux qu'ils auraient pu être, dans une économie appliquant les prix internationaux. Cette conclusion vient consolider l'approche du prix non rémunérateur des activités de la production. Avec l'hypothèse de la constance des prix, les mesures de politique n'ont pas d'impact sur cet indicateur.

Nous concluons que nos paysans ici ne bénéficient pas de mesures de protection par rapport à l'importation de riz.

Le NPI (Coefficient de protection nominale des intrants échangeables) a une valeur supérieure à 1 , soit 1,05 . Cela indique un prix domestique des intrants échangeables ( NPK, UREE,..) supérieur au prix international. On pourrait alors relever l'existence de politiques nationales (taxes directes et indirectes) défavorisant les producteurs de riz local, pour l'acquisition d'intrants échangeables, aussi bien en situation de référence qu'avec la SNDR.

Enfin, le CPE (coefficient de protection effective) indique le degré réel d'incitation par l'effet combiné des politiques de prix du riz local et des intrants échangeables utilisés dans son processus de production. Il reste inférieur à 1 pour les deux périodes que nous comparons (0,56 pour l'option1 et 0,57 pour l'option2). Cela signifie que la combinaison des taxes sur le riz local produit, et les biens échangeables, résulte en une distribution effective des revenus inférieurs à ce qu'elles seraient en cas d'application, toutes 
choses égales par ailleurs, aux prix internationaux. Ainsi, cette activité fait l'objet de taxation. Par ailleurs, on observe une dégradation de l'incitation à produire chez nos paysans et cela malgré la mise en œuvre de la SNDR.

* Riz irrigué ou riz de bas-fond aménagé avec maîtrise totale d'eau

Tableau 6 : MAP avec la SNDR pour le système de riz irrigué

Recettes (en Intrants échangeables Domestiques (en Bénéfice (en

Prix du

FCFA) (en FCFA) FCFA) FCFA)

marché

1027500

68219

158175

801106

Prix de

référence

1265230

64912

153966

1046352

Divergences

$-237730$

3307

4209

$-245246$

$\begin{array}{ll}\mathrm{RCBF}=0,16 & \mathrm{CRI}=0,13 \\ \mathrm{RCBE}=0,17 & \mathrm{CR}=0,77\end{array}$

$\mathrm{CPN}=0,81 \mathrm{NPI}=$

1,05

$\mathrm{CPE}=0,8$

$\mathrm{TSP}=-0,19$

$\mathrm{ESP}=-0,24$

\section{Indicateurs MAP statu quo ou MAP après la crise post- électorale}
$\mathrm{RCBF}=0,45 \quad \mathrm{RCBE}=0,31$
$\mathrm{CRI}=0,24$
$\mathrm{CR}=0,40$
$\mathrm{CPN}=0,59$
$\mathrm{CPE}=0,55$
$\mathrm{TSP}=-0,42 \mathrm{ESP}=-0,7 \quad \mathrm{NPI}=1,05$
Source : calcul de l'auteur
Interprétation du tableau precedent

\begin{tabular}{|c|c|}
\hline $\begin{array}{l}\text { Points de ressemblance avec les } \\
\text { indicateurs de compétitivité du } \\
\text { système RP }\end{array}$ & $\begin{array}{l}\text { Points de divergence avec les indicateurs de } \\
\text { compétitivité du système RP }\end{array}$ \\
\hline $\begin{array}{l}\text { - Les indicateurs sont tous } \\
\text { inférieurs à 1, donc même } \\
\text { commentaire : activité } \\
\text { rentable du double point de } \\
\text { vue financier et économique. }\end{array}$ & $\begin{array}{l}\text { - Ce système utilise les ressources domestiques } \\
\text { avec une rationalité économique de } 76 \% \text { en } \\
\text { option } 1 \text { et de } 87 \% \text { en option } 2 \text {. D'où une nette } \\
\text { amélioration de la compétitivité avec la SNDR; } \\
\text { - La capacité d'autofinancement des producteurs } \\
\text { se traduit par un RCBF }=0,45 \text { en option } 1 \text { et une } \\
\text { nette baisse en option } 2 \text { avec une valeur de } 0,16 \text {. } \\
\text { Ainsi, nous avons aussi une importante } \\
\text { progression de la rentabilité des producteurs avec } \\
\text { la SNDR. }\end{array}$ \\
\hline $\begin{array}{l}\text { Points de ressemblance avec les } \\
\text { indicateurs } \\
\text { économiques du système } R P\end{array}$ & $\begin{array}{l}\text { Points de divergence avec les indicateurs d'incitations } \\
\text { économiques du système RP }\end{array}$ \\
\hline $\begin{array}{l}\text { - Les indicateurs sont tous } \\
\text { inférieurs à } 1 \text {, donc même } \\
\text { commentaire : activité sujet à } \\
\text { une taxation ; } \\
\text { - } \quad \text { Même valeur du NPI = } 1,05 \text {. }\end{array}$ & $\begin{array}{l}\text { - Le CPN passe de } 0,59 \text { en option } 1 \text { à } 0,81 \text { en } \\
\text { option 2. D'où l'atténuation du manque de } \\
\text { mesures de protection du paddy avec la SNDR } \\
\text { puisque } 0,81 \text { est proche de } 1 \text {; } \\
\text { - Le CPE passe de } 0,55 \text { en option } 1 \text { à } 0,8 \text { en option } \\
\text { 2. Ainsi, on devrait espérer une baisse plus } \\
\text { importante de la taxation de cette activité avec la } \\
\text { SNDR. }\end{array}$ \\
\hline
\end{tabular}




\section{Analyse de sensibilité}

Le but de cette partie est de montrer la sensibilité c'est à dire le sens et l'allure de l'évolution entre d'une part le prix paritaire, le degré de surévaluation du FCFA, le prix des intrants, le coût de la main d'œuvre, le prix au producteur et le rendement et la performance économique (RCBF et $\mathrm{CRI}$ ); et d'autre part entre ces mêmes variables et les indicateurs d'incitation économique (CPN, CPE, ESP). En effet il est évident que la rentabilité et la compétitivité économique sont indissociables de l'environnement économique constituant le cadre incitatif dans lequel opère le producteur. Ce cadre lui-même détermine la nature et la structure des transferts implicites qui se font entre le secteur agricole et le reste de l'économie.

Nous accordons un grand intérêt au seuil critique correspondant à 1 (un) pour les indicateurs que nous avons retenus parce que l'interprétation à partir de ce seuil permet de dire toutes choses étant égales par ailleurs, à quel moment un système initialement rentable ou compétitif cesse de l'être et vice versa; ainsi que la nature des incitations implicites qui sont en vigueur.

La démarche est basée sur la statique comparative qui consiste à faire varier un seul facteur tandis que les autres sont maintenus constants. Mais, cette démarche s'effectuera à partir de la matrice d'analyse de politique de l'ensemble des systèmes de production du riz local en Côte d' Ivoire.

Par ailleurs, le choix des intervalles de variation, bien que n'ayant pas été retenus sur la base d'une projection rigoureuse, n'est pas préjudiciable à notre avis à la qualité des résultats; étant entendu que l'objectif est de déterminer: (a) la nature (positive ou négative) de la relation entre les variables et (b) le "point de rupture" ou seuil pour chacune des variables ciblées. Enfin, cette démarche nous permettra par la suite de tester deux hypothèses :

H 1 : Les politiques d'intervention directes (prix des intrants et prix au producteur) sont celles qui influencent le plus la performance des systèmes de production.

H 2 : Une politique visant à accroître les rendements agricoles est de nature à améliorer la "compétitivité globale" (rentabilité financière et avantage comparatif) des systèmes de production sans occasionner un surcoût.

\section{Prix paritaire de référence du riz paddy et indicateurs}

L'impact de l'augmentation du prix paritaire du paddy sur les indicateurs de compétitivité est illustré par le graphique 1 suivant qui indique une corrélation négative entre ces variables. 


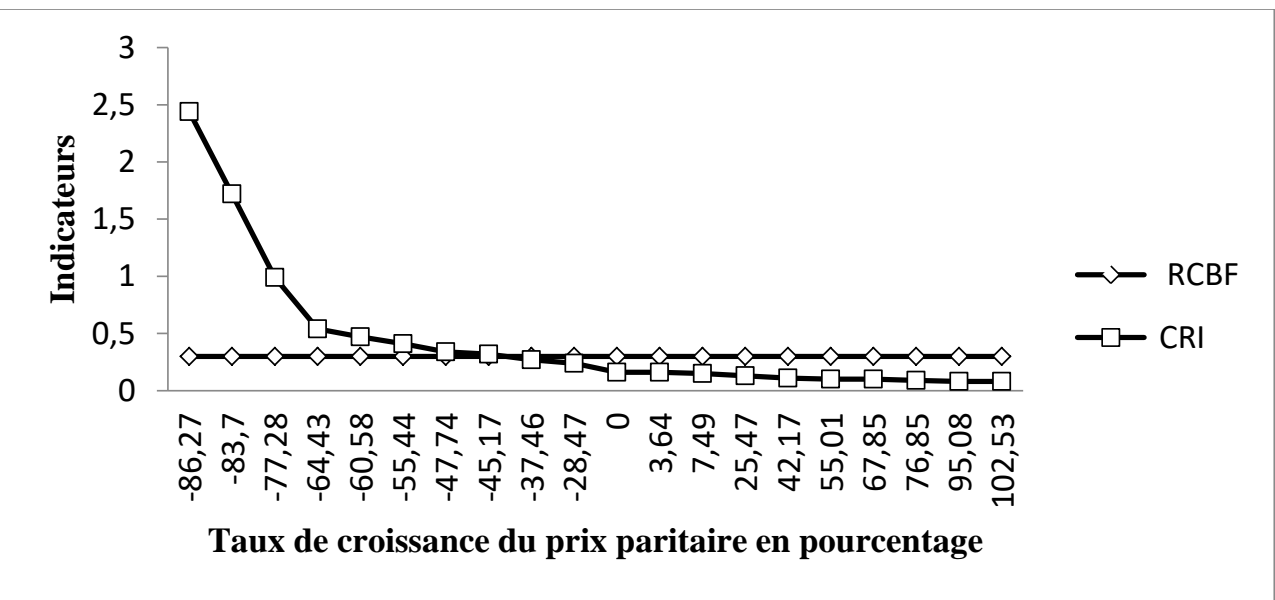

Graphique 1 : Sensibilité des indicateurs de compétitivité au prix paritaire de référence Source : l'auteur

En effet, il existe une relation inverse entre le coût en ressources intérieures et le prix paritaire tandis qu'il n'existe pas de lien entre cette variable et le ratio coût bénéfice financier, car le prix au producteur de riz n'est pas directement dépendant de ce prix.

Le seuil pour le CRI est atteint lorsque le prix paritaire est de 49660FCFA/tonne de riz blanchi; cela correspond à une baisse du prix paritaire de 77,36\%. Au-delà de ce prix la rentabilité économique de la production rizicole est assurée.

Quant à l'effet de l'augmentation du prix paritaire de référence sur la structure des incitations, il est décrit par le graphique 2 suivant. Ce graphique indique qu'il y a une relation inverse entre le prix paritaire et les indicateurs d'incitation économique. Ainsi lorsque ce dernier augmente, ceux-ci baissent.

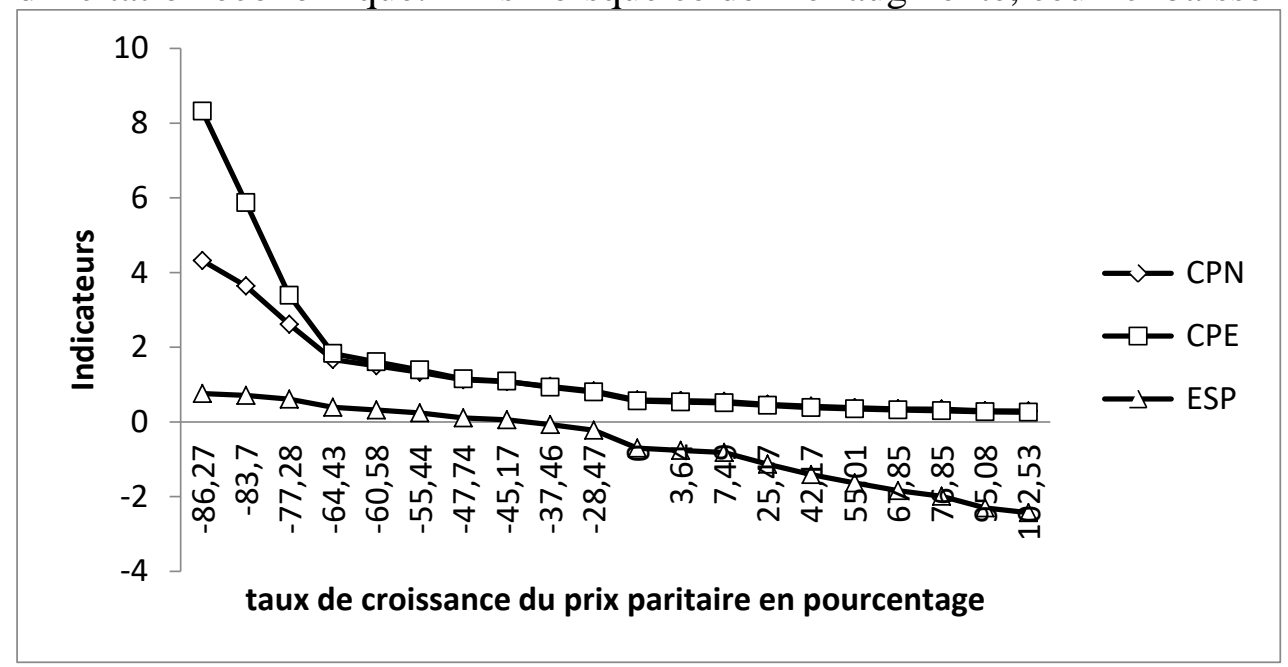

Graphique 2 : Sensibilité des indicateurs d’incitation économique au prix paritaire de référence Source : l’auteur 
Le seuil critique pour le coefficient de protection nominale (CPN) et le coefficient de protection effective (CPE) correspondant à l'unité c'est à dire la situation dans laquelle le produit et les intrants ne sont ni taxés ni subventionnés se situent à 192310FCFA/tonne de riz blanchi importé soit une variation de $-40,72 \%$. Ainsi, nous pouvons reformuler le résultat de Koné (op cit) au sujet de la corrélation entre le prix paritaire et les indicateurs d'incitation économique: "lorsque la situation sur le marché international à tendance à générer un prix assez bas, la production nationale est de plus en plus protégée car elle devient de moins en moins compétitive comme il a été noté plus haut ». Lorsque la compétitivité s'améliore, la part des transferts dans les revenus des producteurs baisse également comme on le voit à travers l'équivalent subvention au producteur (ESP).

\section{Rapport des taux de change et indicateurs}

Comme nous le constatons sur le graphique 3 suivant, le rapport des taux de change (degré de distorsions) n'a pas de rapport avec le ratio coût bénéfice (RCBF) dont la valeur est estimée à 0,3 alors que le ratio du coût en ressources intérieures décroît avec le taux de change.

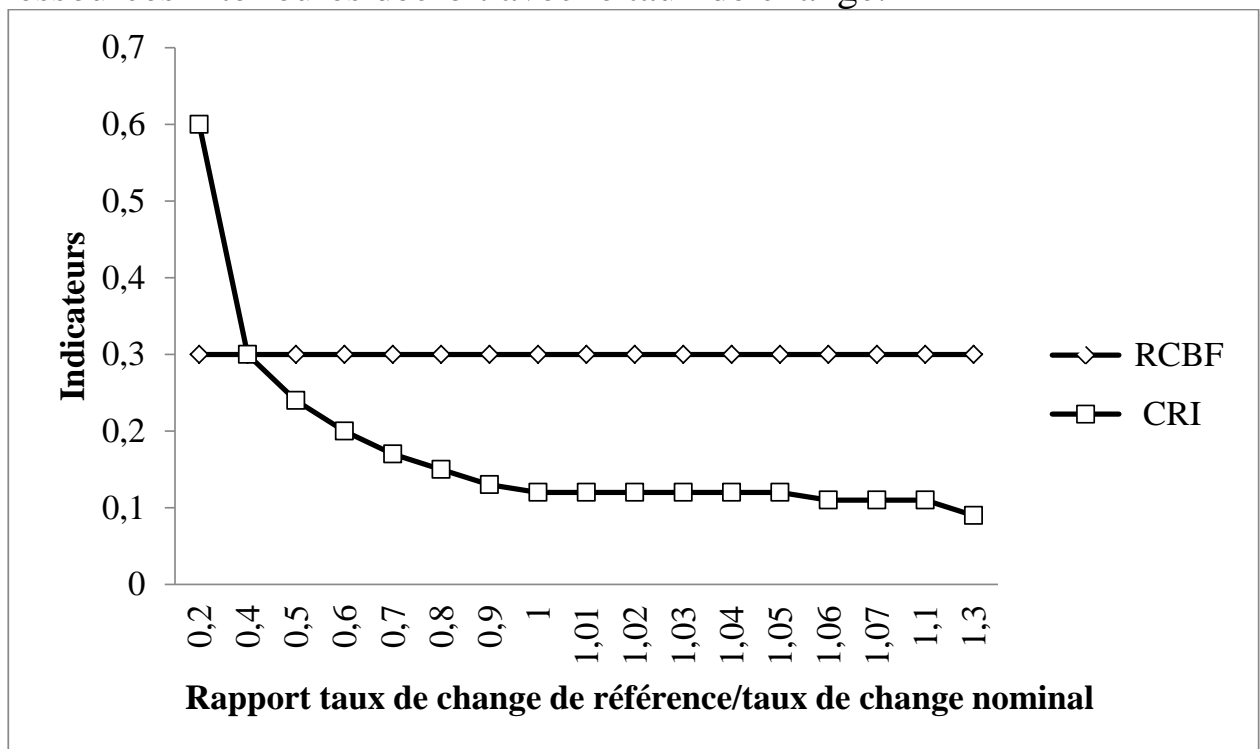

Graphique 3 : Sensibilité des indicateurs de compétitivité économique du paddy au rapport du taux de change

Lorsque le rapport des taux croît, ce qui correspond à une surévaluation du F CFA par rapport au Dollar, la compétitivité internationale du riz domestique s'améliore car le coût en ressources intérieures baisse. Ainsi, la compétitivité du riz local augmente avec la surévaluation du FCFA. Il s'agit d'une situation paradoxale avec un riz importé de plus en plus coûteux qui entraînerait la chute des importations. L'explication du paradoxe 
vient du fait que la structure actuelle du marché du riz consommé est très défavorable aux riziculteurs qui gagnent des revenus très marginaux par rapport aux autres acteurs de la filière. Une surévaluation du FCFA contribuerait à retrouver leur compétitivité perdue amené par l'environnement économique défavorable.

Cette appréciation de la monnaie locale a également des répercussions sur la nature des incitations offertes aux producteurs. Cela est présenté sur le graphique 4 suivant qui met en relation le rapport des taux de change et les indicateurs d'incitation économique.

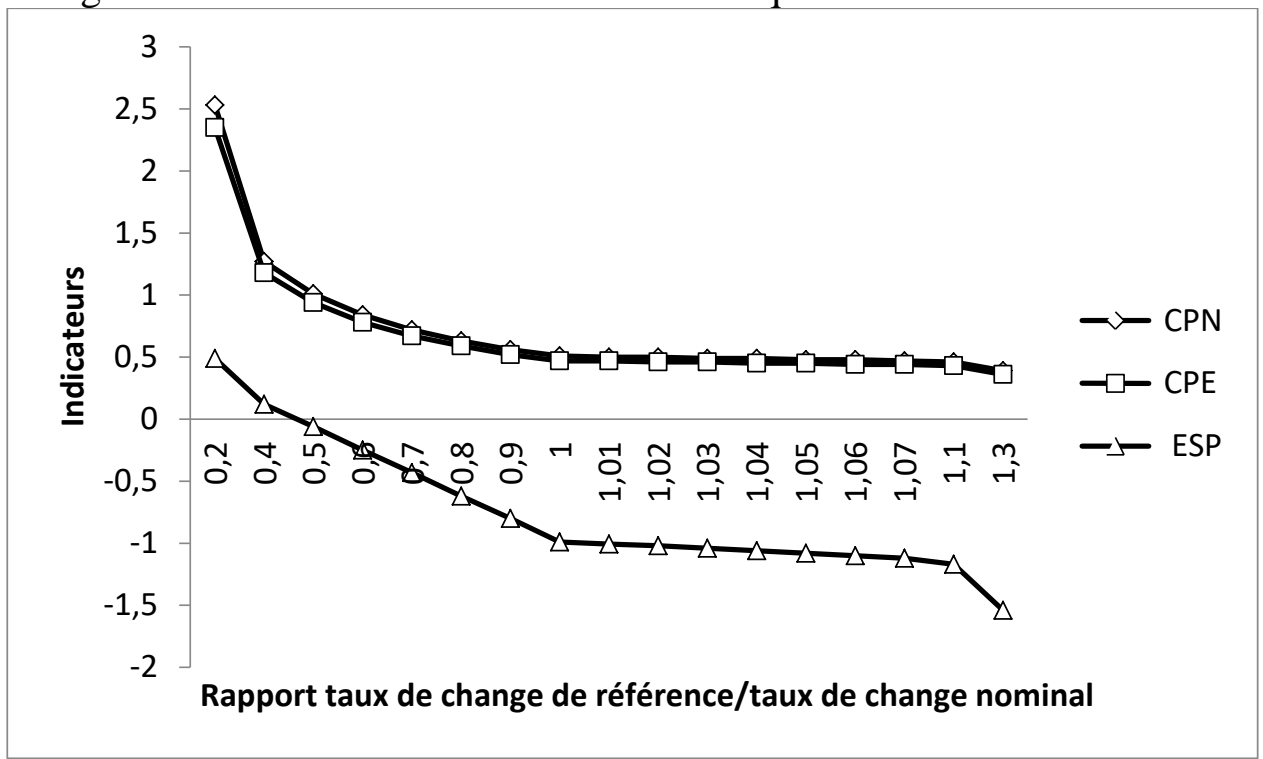

Graphique 4 : Sensibilité des indicateurs d’incitation économique du paddy au rapport du taux de change

Comme précédemment, la surévaluation du FCFA par rapport au Dollar diminue la protection accordée aux producteurs du fait que son impact sur les intrants échangeables importés qui sont implicitement subventionnés est négligeable dans le coût total de production dominé par les intrants non échangeables. Par ailleurs, les revenus au prix de référence nettement supérieur à ceux au prix du marché s'accroissent davantage avec la surévaluation du FCFA. La surévaluation induit donc une absence de protection accrue pour les producteurs de paddy dont la structure des coûts de production est dominée par les intrants non échangeables et un prix économique du paddy de 253,046 FCFA/kg très au dessus du prix financier fixé à 150 FCFA/kg.

\section{Coût moyen pondéré de la main d'œuvre et indicateurs}

Dans le cadre de notre étude, il faut noter que les coûts de la production de riz sont essentiellement des frais liés à la main-d'œuvre. Les 
coûts des autres facteurs de production sont généralement plus faibles. Dans l'ensemble, la main-d'œuvre qui est souvent non salariée est quasi exclusivement d'origine familiale, elle est la plus importante source de travail.

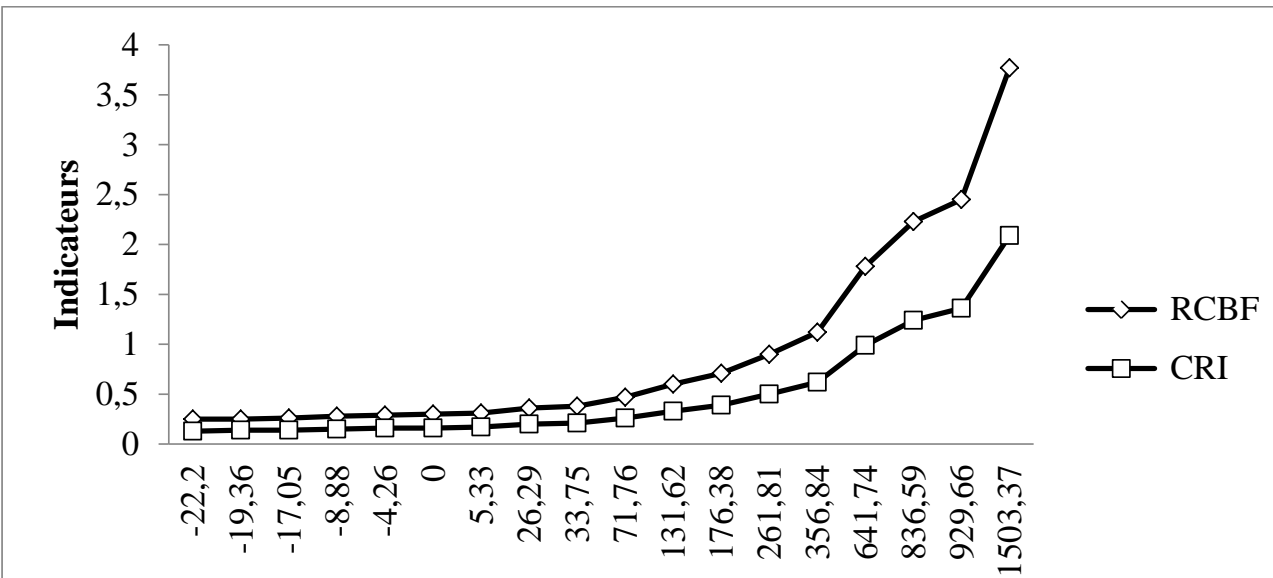

taux de croissance du coût moyen pondéré de la main d'oeuvre en pourcentage

Graphique 5 : Sensibilité des indicateurs de compétitivité au salaire de la main d’œuvre agricole Source : l'auteur

Le graphique 5 précédent, permet de construire la corrélation qui existe entre le coût de la main d'œuvre et les indicateurs de compétitivité. L'allure croissante des courbes indique clairement une relation directe entre ces variables. Par ailleurs, ce graphique montre que la rentabilité financière est plus influencée par le coût de la main d'œuvre que la rentabilité économique puisque la courbe de RCBF est au dessus de la courbe de CRI.

Au-delà de 4220 FCFA par jour soit une variation de 649,56\%, la production de paddy n'a plus d'avantage comparatif tandis qu'elle n'est plus rentable dès que le coût moyen pondéré journalier de la main d'œuvre excède 2280 F CFA/jour qui correspond à une variation 304,97\%. Il est donc évident que la compétitivité financière des systèmes de production traditionnels de paddy est plus sensible au coût de la main d'œuvre que ne l'est la rentabilité sociale. Ce coût est donc déterminant pour le profit financier des riziculteurs en Côte d'Ivoire.

Les implications de la variation du coût moyen pondéré de la maind'œuvre agricole sur les incitations en direction du système de production sont analysées à l'aide du graphique 6 ci-dessous. 


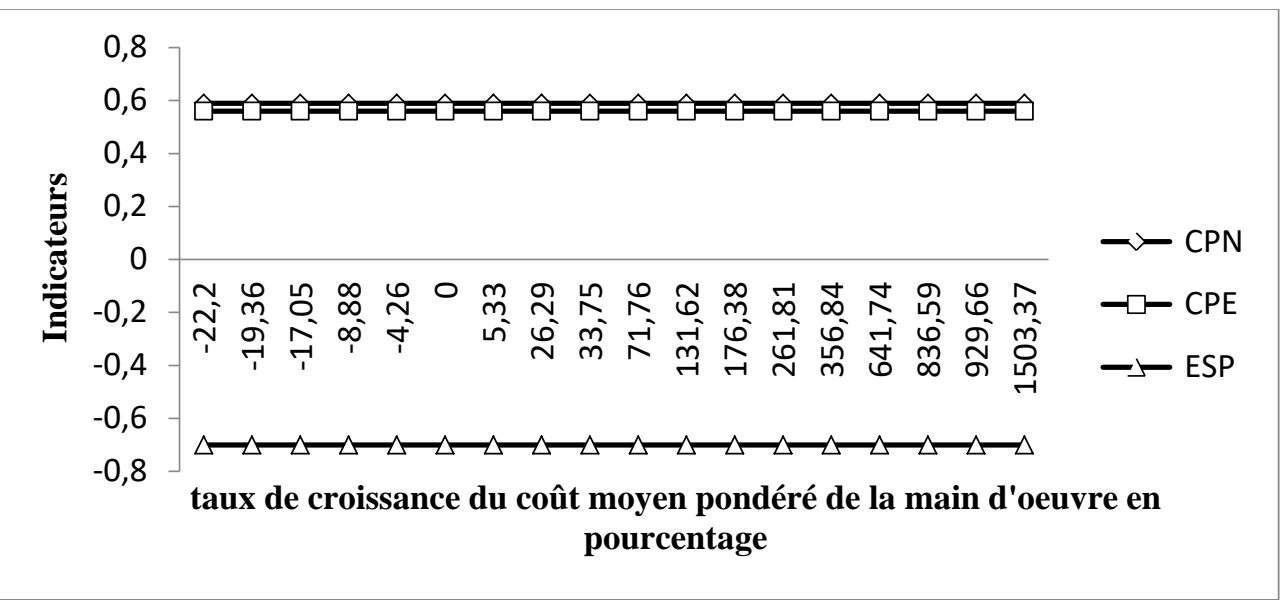

Graphique 6 : Sensibilité des indicateurs d'incitation économique au salaire de la main d'œuvre agricole Source : l'auteur

Comme cela apparaît sur le graphique, le niveau du coût journalier de la main d'œuvre agricole (le salaire de l'employé agricole) n'a pas d'incidence sur la structure des incitations. On remarque qu'aucun de ces indicateurs ne varie quelque soit le salaire agricole. L'explication est qu'en réalité les pouvoirs publics n'interviennent pas directement sur le marché du travail agricole.

\section{Coût des intrants chimiques et indicateurs}

Sur 895 personnes enquêtées, 609 ont affirmé utiliser les engrais dans la production de riz. Pour ceux qui ne les utilisent pas (286 personnes), la raison principale est la cherté de ces produits. Ainsi, notre étude montre que la majorité des paysans font usage de l'engrais dans la culture du riz. L'incidence du renchérissement des intrants chimiques sur les indicateurs de compétitivité est décrite par le graphique 7 suivant.

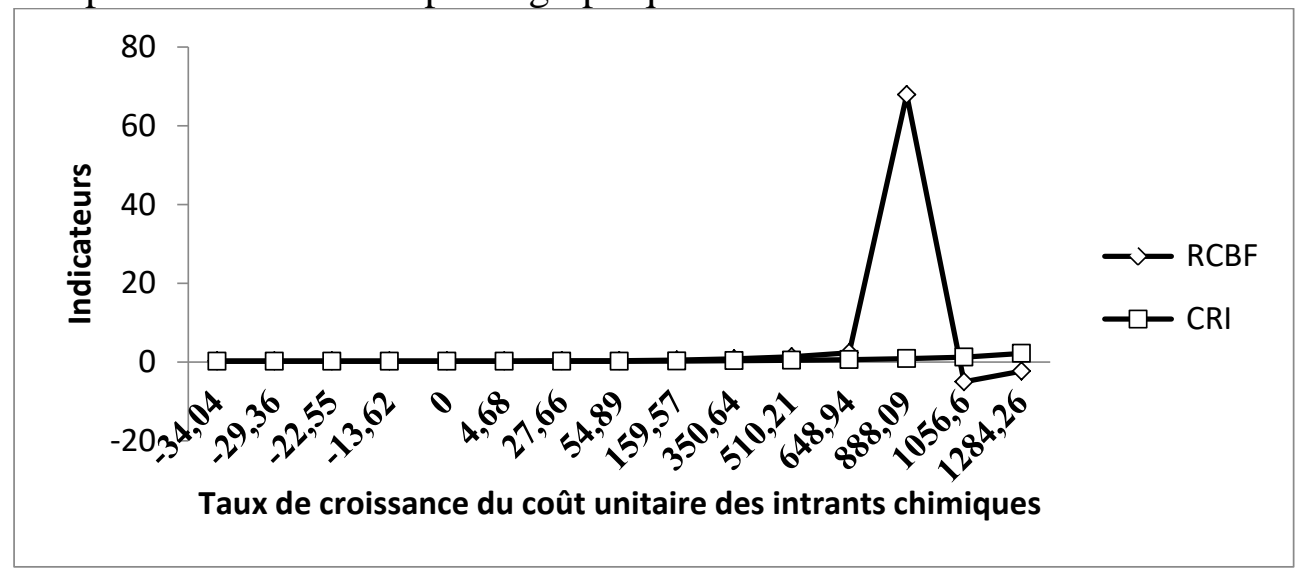

Graphique 7 : Sensibilité des indicateurs de compétitivité au coût moyen unitaire des intrants chimiques (engrais et herbicides)

Source : l'auteur 
Au niveau de l'avantage comparatif (compétitivité extérieure), le graphique 7 précédent montre que la courbe de CRI est très peu sensible aux coûts des intrants chimiques. La production demeure rentable malgré des niveaux élevés du prix des intrants.

Tandis que pour la rentabilité privée (courbe de RCBF), le seuil est plus vite atteint comparativement à celui de la compétitivité extérieure. Ceci montre que la rentabilité des riziculteurs dépend dans une grande mesure du prix des intrants. Cela s'explique par le fait que les intrants chimiques sont utilisés dans des proportions importantes par la majorité des riziculteurs.

L'accroissement du coût des intrants chimiques ne modifie que le coefficient de protection effective (CPE) dans la structure des incitations comme il apparaît sur le graphique 8 suivant.

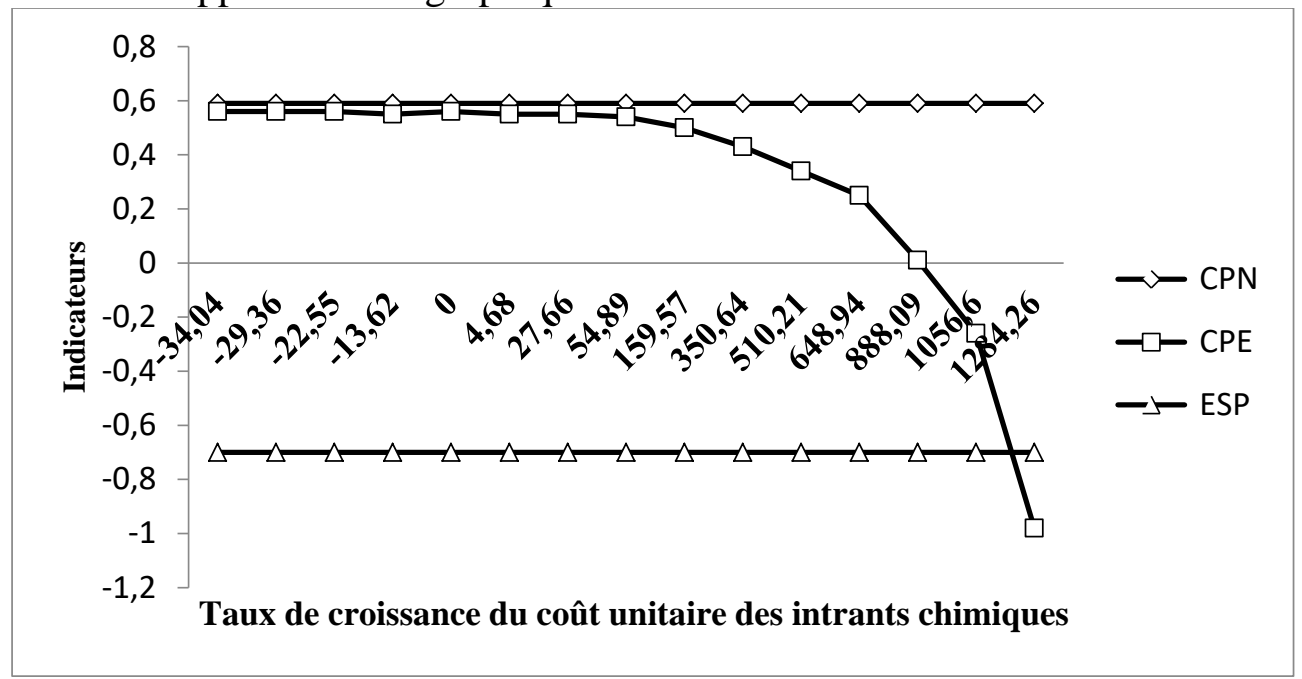

Graphique 8 : Sensibilité des indicateurs d'incitation économique au coût moyen unitaire des engrais et herbicides

Source : l'auteur

L'effet d'une hausse du coût des intrants n'est perceptible que sur le coefficient de protection effective (CPE) à cause principalement de la composition des intrants chimiques : les intrants chimiques même s'ils sont produits localement incorporent des composantes échangeables. En situation de renchérissement du coût de ces intrants, il n'y a pas de transferts implicites en direction des producteurs, seule s'impose une protection sur les facteurs de production.

\section{Prix au producteur et indicateurs}

L'impact du prix au producteur du paddy sur la rentabilité financière et l'avantage comparatif est analysé à partir du graphique 9 ci-dessous. 


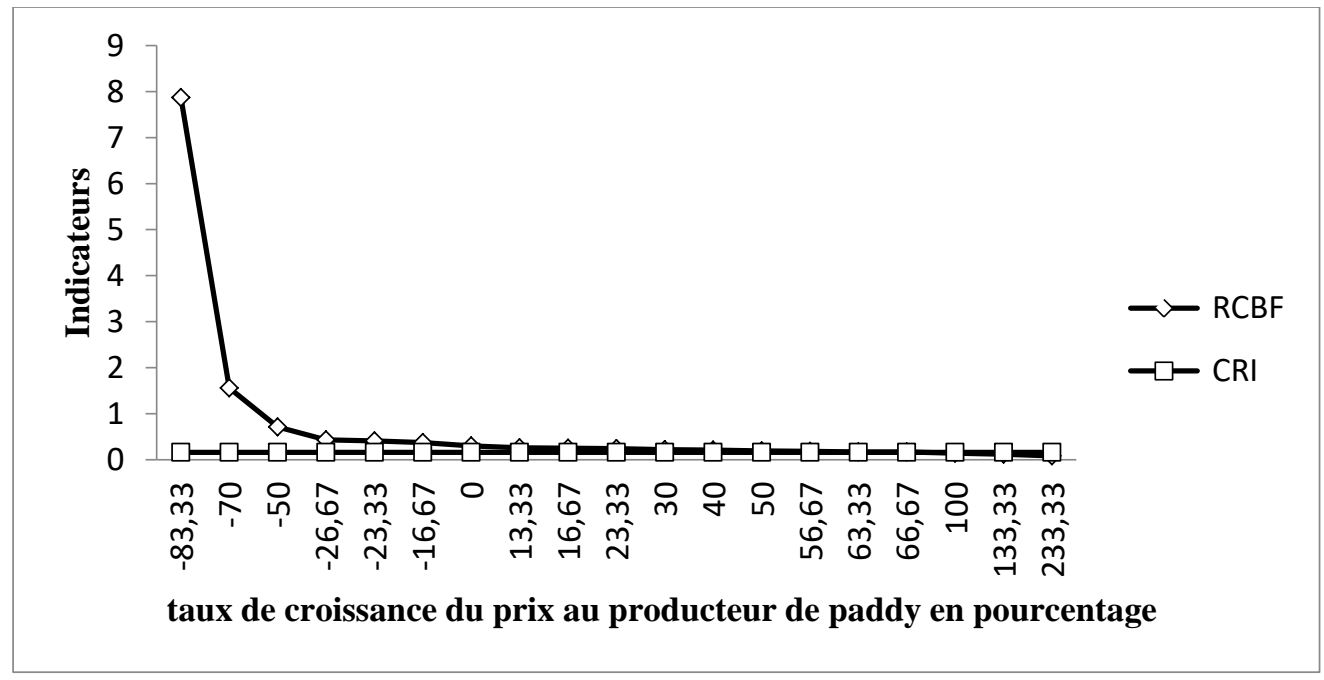

Graphique 9 : Sensibilité des indicateurs de compétitivité au prix au producteur Source : l'auteur

On remarque que cette croissance du prix au producteur n'a aucune incidence sur le coût en ressources intérieures (CRI) qui demeure constant parce que le prix au producteur n'est pas directement lié au prix international. L'augmentation (ou la baisse) du prix au producteur ne contribue pas à améliorer la compétitivité extérieure du riz local. La politique du prix au producteur a donc un effet neutre sur la compétitivité extérieure de la production rizicole à court terme. L'une des explications est aussi le fait que le coût en ressources est mesuré à l'aide du prix paritaire à l'importation qui n'est pas lié au prix au producteur.

Bien que cette politique n'ait pas d'impact sur la compétitivité de la production locale de paddy, son impact sur la rentabilité financière est assez important comme on peut le voir sur le même graphique. En effet plus le prix effectivement payé au producteur est élevé, plus l'activité de production devient rentable à travers la baisse soutenue du ratio coût bénéfice financier (RCBF).

Malgré le fait qu'elle n'a pas d'impact sur la compétitivité extérieure du paddy, la hausse du prix au producteur accroît plus ou moins vite les niveaux des transferts au profit des producteurs comme le montre le graphique 10 suivant. 


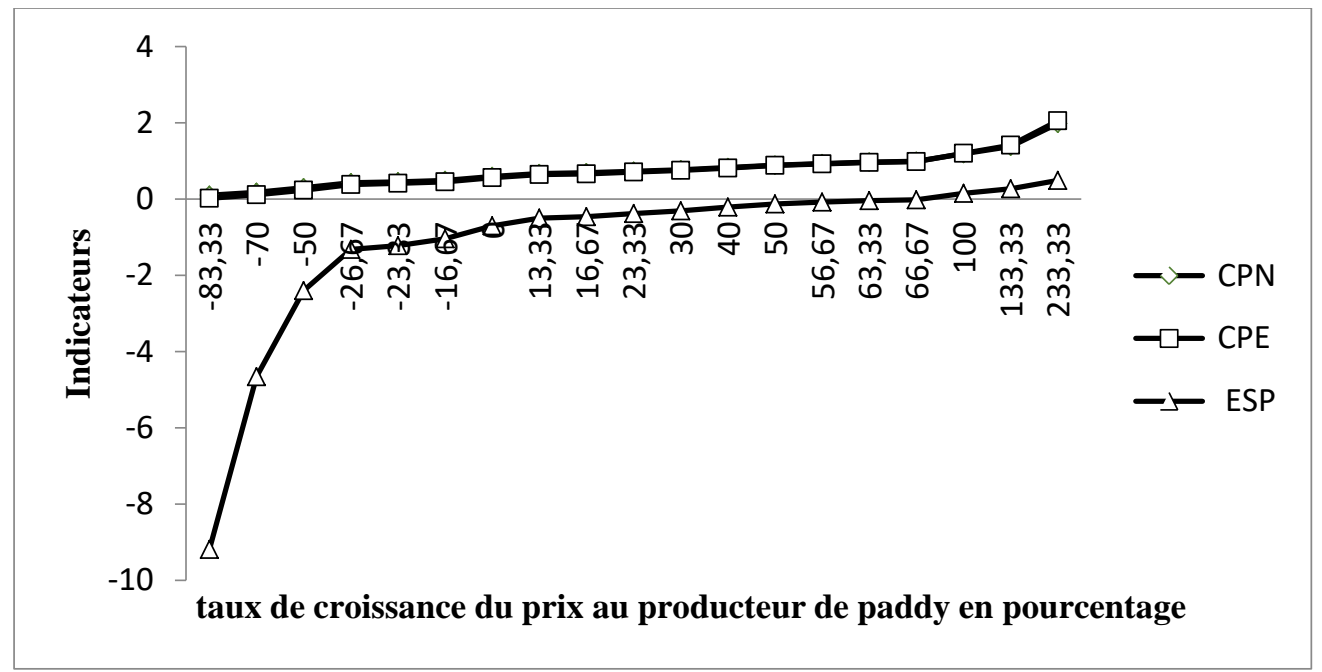

Graphique 10 : Sensibilité des indicateurs d'incitation économique au prix au producteur Source : l'auteur

La protection implicite et non implicite accordées au producteur croissent moins vite avec la hausse du prix au producteur mais donnent lieu à des courbes presque confondues. Au-delà de $250 \mathrm{~F} \mathrm{CFA/Kg}$ de paddy, la protection s'impose pour l'ensemble (le produit et les facteurs) et pour le paddy uniquement.

\section{Rendement du producteur et indicateurs}

Le rendement du paddy joue un rôle très important dans la compétitivité et la rentabilité de l'activité de production. Le graphique 11 ciaprès établit le lien entre les rendements et les indicateurs de compétitivité de la production rizicole.

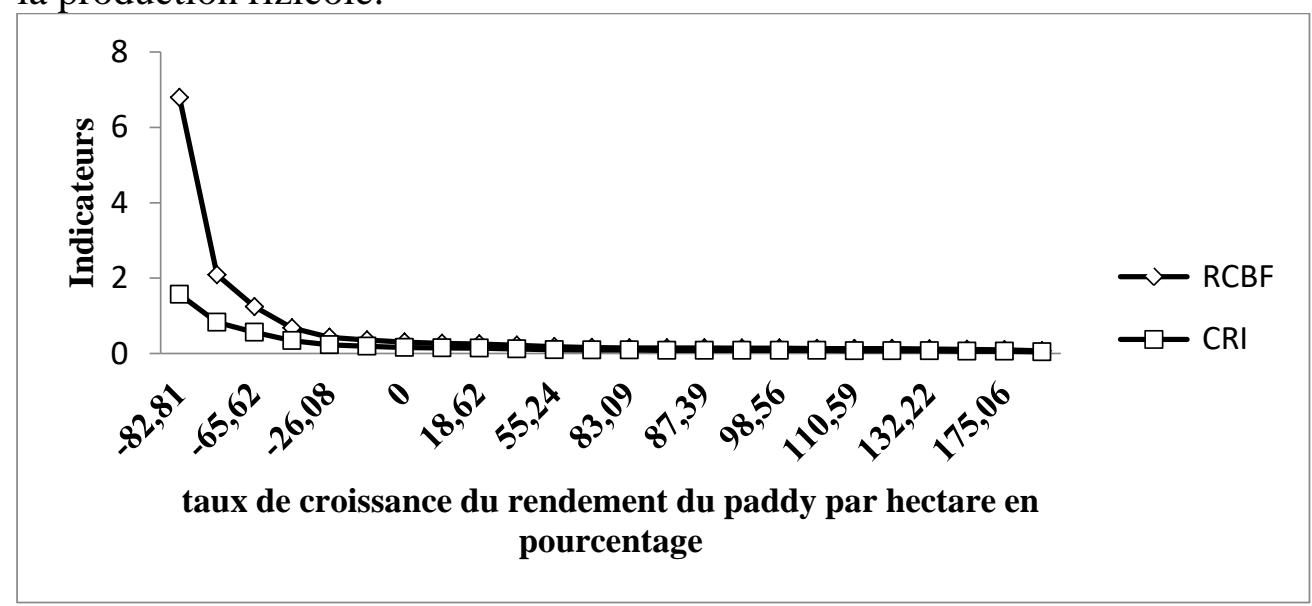

Graphique 11 : Sensibilité des indicateurs de compétitivité au rendement du producteur Source : l'auteur 
La relation entre les rendements et les indicateurs de compétitivité met en évidence le fait que l'amélioration des rendements de paddy exerce une forte influence sur ces derniers. Le seuil de rentabilité financière est de $1220 \mathrm{Kg} / \mathrm{Ha}$ et de $695 \mathrm{Kg} / \mathrm{Ha}$ pour l'avantage comparatif. Ces rendements correspondent respectivement à une baisse de 58,05\% dans le premier cas et une baisse de 76,10\% dans le second. Il est donc évident que les producteurs tirent moins avantage de l'amélioration des rendements que la collectivité. Ce résultat indique donc que la croissance des rendements est un facteur qui accroît à la fois les profits privés et la compétitivité de la production rizicole.

Au niveau de la structure des incitations, le graphique 12 ci-après met en relation le rendement et les indicateurs d'incitation économique.

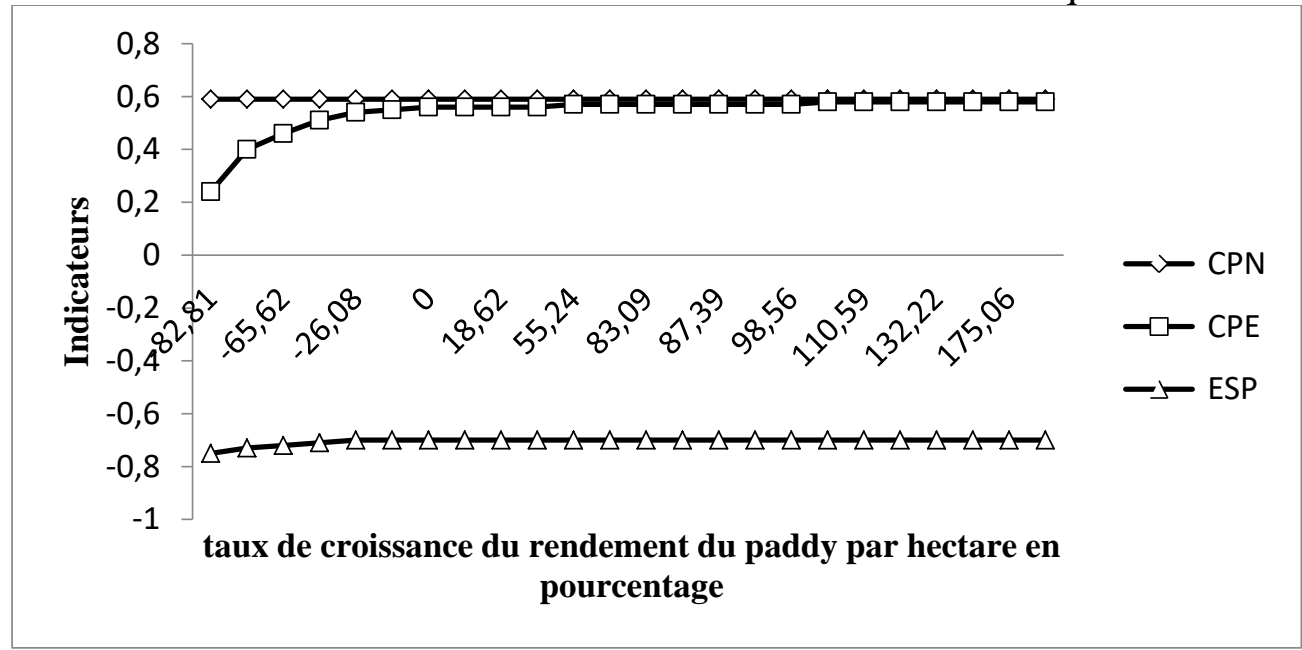

Graphique 12 : Sensibilité des indicateurs d'incitation économique au rendement du producteur

Source : l'auteur

L'analyse montre qu'un accroissement des rendements n'implique pas un transfert supplémentaire de ressources en direction des producteurs de paddy; le coefficient de protection nominale (CPN) et l'équivalent subvention au producteur (ESP) restent constants. De plus l'augmentation du rendement de paddy entraîne une absence de protection sur les intrants telle que cela apparaît à travers des valeurs du coefficient de protection effective (CPE) inférieures à 1 même si cet indicateur nous donne une courbe croissante.

\section{Test des hypothèses 1 et 2}

\section{Test de l'hypothèse 1}

Cette hypothèse stipule que les politiques d'intervention directes (prix des intrants et prix au producteur) sont celles qui influencent le plus la performance des systèmes de production. 
L'hypothèse n'a pas été vérifiée car toutes les variables de notre analyse de sensibilité ont un impact perceptible sur le ratio de coût en ressources intérieures (CRI) à l'exception du prix au producteur. Il est donc apparu que seule la politique d'intervention (politique des intrants) a un impact sur l'avantage comparatif contrairement à celle du prix au producteur qui a un effet nul.

\section{Test de l'hypothèse 2}

Elle stipule que seule une politique visant à accroître les rendements agricoles est de nature à améliorer la "compétitivité globale" (rentabilité financière et avantage comparatif) des systèmes de production sans occasionner un surcoût.

Cette hypothèse a été vérifiée au cours de cette analyse de sensibilité. En effet parmi toutes les variables que nous avons retenues (elles ne sont pas exhaustives), il n'y a que l'amélioration du rendement agricole qui améliore à la fois tous les indicateurs de compétitivité (RCBF et CRI).

\section{Conclusion et recommandations}

In fine, il ressort de cette étude que la SNDR (2012-2020) améliorera la compétitivité extérieure de même que les profits financiers des paysans des systèmes de production en Côte d'Ivoire. Mais, concernant le protectionnisme de la culture du riz, on note avec cette SNDR, une dégradation des incitations à produire dans le système de riz pluvial alors qu'une amélioration de la protection du système irrigué est imminente.

L’analyse de sensibilité vient renforcer ce précédent développement sur l'impact de la SNDR sur la production de riz dans la mesure où elle vise à apprécier les changements de la compétitivité des producteurs par rapport à certaines variables clées avec 2012 comme année de base. Les résultats obtenus de cette simulation se présentent comme suit :

$\checkmark \quad$ L'accroissement du rendement a été identifié comme le seul facteur capable

d'améliorer à la fois la rentabilité financière, la compétitivité extérieure (avantage comparatif), en réduisant par la même occasion le soutien (transferts) aux producteurs. Ce résultat indique donc que l'accroissement du rendement agricole devrait être un objectif prioritaire en matière de politique agricole. Pour cela, il faut promouvoir la recherche des variétés à haut rendement, ainsi que la vulgarisation et l'encadrement pour l'adoption de celles-ci.

$\checkmark \quad$ Les analyses de sensibilité ont mis en évidence le rôle paradoxale du taux de change

réel dans la compétitivité des productions agricoles. En effet, la compétitivité du riz local augmente avec la surévaluation du FCFA. Il s'agit d'une situation paradoxale dans laquelle le riz importé deviendrait de plus en plus 
coûteux entraînant la chute des importations. Ce qui est sans doute un déni de la réalité. L’explication du paradoxe vient du fait que la structure actuelle du marché du riz consommé est très défavorable aux riziculteurs qui gagnent des revenus très marginaux par rapport aux autres acteurs de la filière. Une surévaluation du FCFA contribuerait à retrouver leur compétitivité perdue amené par l’environnement économique défavorable.

$\checkmark \quad$ Les indicateurs de compétitivité s’améliorent avec une augmentation du prix paritaire, du taux de change réel, du prix au producteur et du rendement agricole; et se détériorent avec l'augmentation des prix des intrants et des facteurs (main d'œuvre).

En ce qui concerne les incitations, elles s'accroissent avec le prix au producteur et ne varient pas assez avec le prix paritaire, le coût de la main d’œuvre, le coût des intrants et le rendement.

\section{References:}

1. FANG, Cheng and Beghin, John C., "Food Self-Suffiency, Comparative Advantage, and Agricultural Trade: A Policy Analysis Matrixfor Chinese Agriculture" (2000). CARD Working Papers. Paper 270. htt://lib.dr.iastate.edu/card_workingpapers/270.

2. KONE, Nouvo, (1998), Efficacité économique, incitations à la production et avantage comparatif des systèmes de production traditionnels du coton et du riz dans le nord-ouest de la Côte d'Ivoire : les zones de Boundiali et d'Odienné, Thèse de Doctorat de 3ème cycle, CIRES, Université d'Abidjan.

3. KOUAKOU B., 2006. "Etude relative à la définition d'une politique de développement et à l'élaboration d'un plan d'action opérationnel". Ministère de l'Agriculture, Filière riz, Rapport définitif. PP 33 - 34.

4. MASTERS, W. A., and A. Winter-Nelson. (1995). "Measuring the Comparative Advantage of Agricultural Activities: Domestic Resource Costs and the Social Cost-Benefit Ratio.” American Journal of Agricultural Economics 77: 243-50.

5. Ministère de l'Agriculture (2007),"Annuaire des Statistiques Agricoles", Direction des Statistiques, de la Documentation et de l'Informatique (DSDI).

6. Mohanty, Samarendu; Fang, Cheng; and Chaudhary, Jagadanand, "Assessing the Competitiveness of Indian Cottn Production: APolicy Analysis Matrix Approach" (2002). CARD Working Papers. Paper 328. htt://lib.dr.iastate.edu/card_workingpapers/328.

7. MONKE, A. Eric et Scott R. PEARSON, (1989), The policy analysis matrix for agricultural development, Ithaca and London: Cornell university press. 
8. ONDR, 2012, STRATÉGIE NATIONALE REVISEEDE DÉVELOPPEMENT DE LAFILIERE RIZ EN CÔTE D'IVOIRE(SNDR) 2012 - 2020, Document de travail, 40 Pages.

9. OUATTARA, Zanga, Fousséni, (2011), Analyse de la compétitivité du riz local en Côte d’Ivoire, Résumé No3-2011-2012, Programme de Renforcement et de Recherche sur la Sécurité alimentaire en Afrique de l'Ouest (PRESAO).

10. SYLLA, Kalilou, (1997), Politiques d'incitation et performance économique des filières café, cacao et riz : le cas du centre ouest de la Côte d'Ivoire, Thèse de Doctorat de 3 ème cycle, CIRES, Université d'Abidjan. 